\title{
Application of SSPSO and SPSO to Optimal Power Flow Solutions along with SSC Device
}

\author{
D. Ganga Bhavani*, N. Rama Narayana and R. Madhusudan \\ 'Department of Electrical and Electronics Engineering, Sir C R Reddy College of Engineering, \\ Eluru - 534007, Andhra Pradesh, India; \\ gangabhavani46@gmail.com, ramanarayana1@gmail.com,sudhankumar266@gmail.com
}

\begin{abstract}
Objectives: In this study, optimization techniques SSPSO and SPSO are used to minimize the total generation cost and active power loss. Methods/Analysis: The power flow analysis gives the information of magnitude of voltages, phase angles and complex power flow at each bus. Optimal Power Flow (OPF) solutions with Static Series Compensator (SSC) FACTS device in the power system to increase the total power transfer. Fast Relative Electrical Distance (F-RED) method based on Simple-Voltage Stability Index (H-index) is used to find the maximum generation supplied to the load. Findings: Comparison was made on SSPSO and SPSO methods and SPSO with SSC gives better results. The H-index is used to judge the power system is stable or unstable. F-RED gives the information, the amount of generators contribution to satisfy the loads. Novelty/ Improvement: SSPSO gives better solution results than SPSO, the methods are applied on IEEE-30 bus system. H- INDEX and F-RED are calculated for the IEEE-5 bus system.
\end{abstract}

Keywords: OPF, SPSO, SSC, SSPSO

\section{Introduction}

Optimal power flow is considered as backbone tool in the power systems. With the increase in demand leads to increase in generation that requires increase in the thermal capacity. For this reason, the problem of Optimal Power Flow $(\mathrm{OPF})^{1}$ is yet to be studied by minimizing the cost, losses andhas becomes the source of concern to secure operation of the power system. The voltage stability limits ${ }^{2,3}$ is also considered for secure operation of the system.

The F-RED ${ }^{4}$ concept is used to know how much amount of generation is generated from each generator to satisfy the load. FACTS are used in power system for controlling power and to increase the transmission capacity near to their thermal limits. FACTS also improve the system stability and security, reduce the reactive power flow, thereby permitting greater active power flow ${ }^{5}$. Different types of FACTS controllers are: a) Series, b) Shunt, c) combination of Series and Shunt.
Nowadays, the new algorithms have evolved as a solution for complex problems. Along with conventional methods intelligent methods like SSPSO and SPSO gives greater convergence characteristics and gives the best global minimum solution.

With the addition of SSC device on the power system forenhancement of voltage stability index by SPSO based power flow solutions was examined in this paper. The proposed SPSO with SSC device was tested on IEEE-30 bus ${ }^{6}$. The results are observed by considering SSC cost also. Comparison analysis was done on the total generation cost and active power losses by optimization methods SPSO and SSPSO for IEEE-30 Bus.

\section{Simple-voltage Stability Index ( $\mathrm{H}$-index)}

The stability of the power system is represented in terms of voltage stability studies. This can be represented with the help of voltage stability index. The scalar magnitude of this 
index can be helpful for monitoring the system parameters. This index-based instability measures and captures unique system behavior in terms of number and interprets them to give the idea of distance to instability. Normal power systems have $\mathrm{H}$-index value lies in between 0 and 1 . For stable operation of the system the $\mathrm{H}$-index should be near to zero. The H-index can be computed with the formula:

$$
H=\left|1-\sum_{n=1}^{g} \bar{F}_{i j} \frac{\bar{V}_{n}}{\bar{V}_{m}}\right|, \mathrm{m}=\mathrm{g}+1 \ldots . ., \mathrm{n}^{1}
$$

where, $\mathrm{H}$ is the simple-voltage stability index

$\bar{V}_{n}$ - is the voltage at the bus $\mathrm{n}$

$\bar{V}_{m}-$ is the voltage at the bus $m$

All the variable sinside the sigma in the above equation are complex quantities. The value $\bar{F}_{i j}$ is calculated from the admittance matrix.

\section{Fast-relative Electrical Distance}

The process of F-RED computes the relative distance between the load and generation buses. Is calculated by using the transmission system parameters. Using this approach, the requirement of generation increase or decrease can be identified. The load shared by different generators acts as function of their relative distance from each of the loads. The reactive power contributed by various sources is also evaluated using this approach.

$$
[\text { F-RED }]=\mathrm{M}-\left[D_{L G}\right]
$$

where, $M$ is the unity matrix of size $L \times G, G$ is the no. of generator buses and $\mathrm{L}$ is the no. of load buses. where, $\left[D_{L G}\right]$ is also known as desired generation proportions matrix

$$
\left[D_{L G}\right]=\operatorname{abs}\left\{\left[F_{L G}\right]\right\}
$$

$\left[D_{L G}\right.$ ] gives the information about the location of the load buses with reference to the generator buses, is widely called as F-RED. The elements of $\left[F_{L G}\right]$ matrix is complex, the columns of $\left[F_{L G}\right]$ represents the no.of generator buses and rows of $\left[F_{L G}\right]$ represents the no.of load buses.

\section{Facts Controllers}

FACTS represent a family of power electronic based devices which are able to improve the power system controllability and stability. The power controllability can be attained by applying the voltage either at midpoint or in series with the line or in phase quadrature with the current flow in the transmission line ${ }^{\mathrm{T}}$.

Static Series Compensator: Series compensators are used to improve the power flow in the line where it is connected.The series capacitive compensation is used to reduce the overall transmission line impedance from sending end to receiving end. SSC also minimizes the possibility of voltage collapse. Representation of SSC is shown in Figure 1; the active and reactive power flow equations when SSC is included between bus ' $m$ ' and ' $n$ ' are:

$$
\begin{aligned}
P_{m n}= & V_{m}^{2} g_{m m}-V_{m} V_{n}\left(g_{m m} \cos \theta_{m n}+b_{m n} \sin \theta_{m n}\right) \\
& -V_{m} V_{s e}\left(g_{m n} \cos \left(\theta_{m}-\theta_{s e}\right)+b_{m n} \sin \left(\theta_{m}-\theta_{s e}\right)\right) \\
Q_{m n}= & -V_{m}^{2} b_{m m}-V_{m} V_{n}\left(g_{m n} \sin \theta_{m n}-b_{m n} \cos \theta_{m n}\right) \\
& -V_{m} V_{s e}\left(g_{m n} \sin \left(\theta_{m}-\theta_{s e}\right)-b_{m n} \cos \left(\theta_{m}-\theta_{s e}\right)\right)
\end{aligned}
$$

The $\mathrm{P}$ and $\mathrm{Q}$ equations from bus ' $\mathrm{n}$ ' to bus ' $\mathrm{m}$ ' are:

$$
\begin{aligned}
P_{n m}= & V_{n}^{2} g_{n m}-V_{m} V_{n}\left(g_{m n} \cos \theta_{n m}+b_{m n} \sin \theta_{n m}\right) \\
& +V_{n} V_{s e}\left(g_{m n} \cos \left(\theta_{n}-\theta_{s e}\right)+b_{m n} \sin \left(\theta_{m}-\theta_{s e}\right)\right) \\
Q_{n m}= & -V_{n}^{2} b_{n n}-V_{m} V_{n}\left(g_{m n} \sin \theta_{n m}-b_{m n} \cos \theta_{n m}\right) \\
& +V_{n} V_{s e}\left(g_{m n} \sin \left(\theta_{n}-\theta_{s e}\right)-b_{m n} \cos \left(\theta_{n}-\theta_{s e}\right)\right)
\end{aligned}
$$

Here

$$
\begin{aligned}
& g_{m n}+j b_{m n}=\frac{1}{Z_{s e}}, g_{m m}=g_{m n}, \\
& b_{m m}=b_{m n}, g_{n n}=g_{m n}, b_{n n}=b_{m n}
\end{aligned}
$$

Operating limits of the SSC:

$$
\begin{gathered}
\mathrm{PE}=\operatorname{Re}\left(V_{s e} I_{n m}^{*}\right)=0 \text { or } \\
-V_{m} V_{s e}\left(g_{m n} \cos \left(\theta_{m}-\theta_{s e}\right)+b_{m n} \sin \left(\theta_{m}-\theta_{s e}\right)\right) \\
+V_{n} V_{s e}\left(g_{m n} \cos \left(\theta_{n}-\theta_{s e}\right)+b_{m n} \sin \left(\theta_{n}-\theta_{s e}\right)\right) t=0
\end{gathered}
$$

The $\mathrm{P}$ and $\mathrm{Q}$ limitations are

$$
\begin{gathered}
P_{n m}-P_{n m}^{\text {specified }}=0 \\
Q_{n m}-Q_{n m}^{s p e c i f i e d}=0
\end{gathered}
$$

Here

$$
P_{n m}^{\text {specified }} \text { and } Q_{n m}^{\text {specified }} \text { are specified "P" and "Q" }
$$

The voltage injected by SSC is $V_{s e} \angle \theta_{s e}$ and the limits are

$$
\begin{aligned}
& V_{s e}^{\min } \leq V_{s e} \leq V_{s e}^{\max } \\
& \theta_{s e}^{\min } \leq \theta_{s e} \leq \theta_{s e}^{\max }
\end{aligned}
$$


where,

$$
\begin{aligned}
& V_{s e}=0.04 \mathrm{p} . \mathrm{u}, V_{s e}^{\min }=0.001, V_{s e}^{\max }=0.2 \\
& \theta_{s e}=87.13^{\circ}, \theta_{s e}^{\min }=90^{\circ}, \theta_{s e}^{\max }=180^{\circ}
\end{aligned}
$$

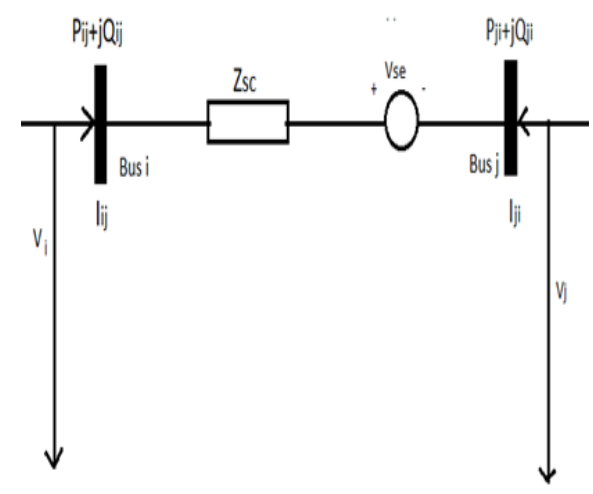

Figure 1. Equivalent circuit of SSC.

\section{Optimal Power Flows}

The SPSO technique with SSC device is applied to optimize the fuel cost of generation and to improve the system performance by maintaining active power and voltage constraints. Mathematically, the OPF objectives are functioning to minimize generation cost and reduce active power losses of the power system.

The objective functions are:

1) Minimization of generation fuel cost

$$
F=\sum_{m=1}^{n g}\left(a_{m} P_{G m}^{2}+b_{m} P_{G m}+c_{m}\right)
$$

To minimize of above objective function subjected to both exact equality and inequality constraints are:

i) Exact-equality constraints:

$$
\begin{gathered}
P_{G m}-P_{D m}-\sum_{n=1}^{n^{1}}\left|V_{m}\right|\left|V_{n}\right|\left|Y_{m n}\right| \cos \left(\theta_{m n}-\delta_{m}+\delta_{n}\right)=0 \\
Q_{G m}-Q_{D m}+\sum_{n=1}^{n^{1}}\left|V_{m}\left\|V_{n}\right\| Y_{m n}\right| \sin \left(\theta_{m n}-\delta_{m}+\delta_{n}\right)=0
\end{gathered}
$$

where, $P_{G m}$ and $Q_{G m}$ are the real and reactive powers at bus ' $m$. The load demand at the bus ' $m$ ' is represented by $P_{D m}$ and, $Q_{D m}$ and the values of $\left|Y_{m n}\right|$ and $\theta_{m n}$ are taken from Y-bus.

ii) Exact-Inequality constraints are:

1. Generators power limits:

$$
\begin{aligned}
& P_{G m}^{\min } \leq P_{G m} \leq P_{G m}^{\max }, m=1, \ldots, N \\
& Q_{G m}^{\min } \leq Q_{G m} \leq Q_{G m}^{\max }, m=1 \ldots N
\end{aligned}
$$

2. Voltage values at every bus:

$$
V_{m}^{\min } \leq V_{m} \leq V_{m}^{\max }, m=1 \ldots N
$$

3. Transformer tap limits:

$$
T_{m}^{\min } \leq T_{m} \leq T_{m}^{\max }, m=1 \ldots N T
$$

4. Reactive power supply due to capacitor banks:

$$
Q_{C m}^{\min } \leq Q_{C m} \leq Q_{C m}^{\max }, m=1 \ldots C S
$$

5. Transmission lines loading limits:

$$
S_{m} \leq S_{m}^{\max }, m=1 \ldots n l
$$

6. Simple voltage stability index limits:

$$
L j_{m} \leq L j_{m}^{\max }, m=1 \ldots N L
$$

iii) FACTS device constraints

$$
\begin{gathered}
V_{s e}^{\text {min }} \leq V_{s e} \leq V_{s e}^{\text {max }} \\
\theta_{s e}^{\text {min }} \leq \theta_{s e} \leq \theta_{s e}^{\text {max }}
\end{gathered}
$$

2) Minimization of total active power loss is

$$
\sum P_{L}=\sum P_{G}-\sum P_{D}
$$

\section{Simplified-particle Swarm Optimization}

Simplified-Particle Swarm Optimization was developed through the imitation of bird flocking or school of fish. It is an evolutionary process, used to obtain the optimal solution for different problems $s^{8-10}$.

The idea of SPSO consists of changing velocity of each particle towards local-best and global-best positions at each iteration. The velocity term is multiplied by a random term, will generate random values towards local best and global best positions. By using method the global best values are obtained very faster way. The other advantage using this method is parameters are few in number (Figure 2) the above The figure represents the searching of particles towards its best position. Each particle modifies its position with changing its velocity using the formula:

$$
\begin{gathered}
v_{m}{ }^{n+1}=w^{*} v_{m}^{n}+c_{1} \operatorname{rand}()_{1}{ }^{*}\left(\text { pbest }_{m}-s_{m}^{n}\right) \\
+c_{2} \operatorname{rand}()_{2}{ }^{*}\left(\text { gbest }-s_{m}{ }^{n}\right)
\end{gathered}
$$

where, $v_{m}^{n}$ is velocity of particle ' $m$ ' at iteration ' $\mathrm{n}$ ', $c_{1}$ and $c_{2}$ are constants, which varies the velocity of a particle towards pbest (local best) and gbest (global best), $\operatorname{rand}_{1}()$ and $\operatorname{rand}_{2}()$ are the numbers generated randomly between zero and one. $s_{m}^{n}$ is current position of particle ' $m$ ' 
at iteration ' $n$ ', pbest ${ }_{m}$ is the local best of ' $m$ ' and gbest is the best value among the local bests of all the particles. The weight function in the above equation can be determined using the below formula.

$$
w=w_{\max }-\left(\left(w_{\max }-w_{\min }\right) /\left(\text { iter }_{\max }\right)\right)^{*} \text { iter }
$$

where, $w_{\max }$ is the final weight, $w_{\min }$ is the initial weight as these limits controls exploration and exploitation of the search space, 'iter ${ }_{\max }$ ' is the total iterations and 'iter' is the present iteration. The positionupdating can be done by using the formula:

$$
s_{m}^{n+1}=s_{m}^{n}+v_{m}^{n+1}
$$

The above procedure is represented in step-by-step manner is shown in Figure 3.

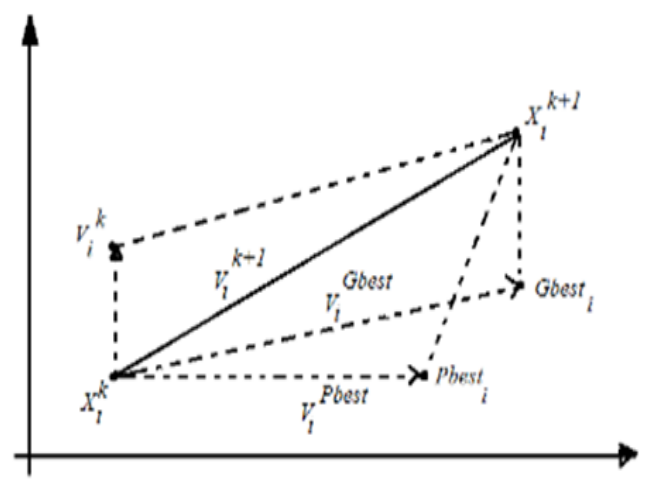

Figure 2. Concept of modification of a searching point by SPSO.

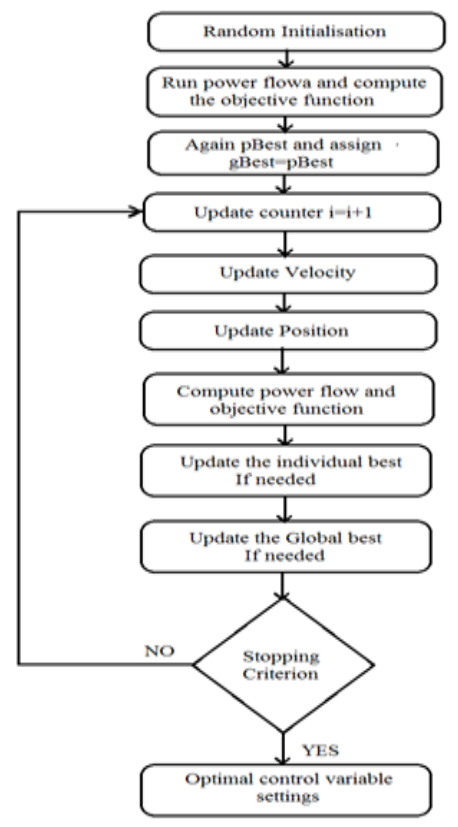

Figure 3. Pictorial representation of SPSO method.

\section{Supervisory-sliced Particle Swarm Optimization}

A new optimization method Sliced Particle Swarm Optimization (SSPSO) is introduces the slicing of search space into rectangular portions. This SSPSO gives the complete solution in terms of reduction in the calculations and tracking of optimal value in each search space.

In SSPSO, the algorithm split the search space into no. of rectangular slotted sections i.e. from outer rectangular slot with high search space towards inner rectangular slot with less search space. The complete solution is obtained by searching all the slices.The comparison among all the slices gives S-best (pbest) and the comparison of S-best of each slices leads to gbest .

Momentum factor (mc) used in the equation (27) with position change gives the convergence and particle velocity changes according position.

The position and velocity updating equations for each particle in each slice are:

$$
\begin{aligned}
& v_{m}{ }^{n+1}=w^{*} v_{m}^{n}+c_{1} \text { rand }_{1}()^{*}\left(\text { pbest }_{m}-s_{m}^{n}\right) \\
& +c_{2} \text { rand }_{2}()^{*}\left(\text { gbest }-s_{m}{ }^{n}\right) \\
& w=\frac{\left(w_{\text {final }}-w_{\text {initial }}\right)\left(T_{\text {iter }}-\text { iter }\right)}{T_{\text {iter }}}+w_{\text {initial }}
\end{aligned}
$$

where, $w_{\text {final }}$ and $w_{\text {initial }}$ are the pre-defined highest and lowest weighting function values, respectively. Larger weighting value gives a global exploration and smaller weightingvalue gives a local exploration.

The current position (new position) can be modified by using the following equation:

$$
s_{m}{ }^{n+1}=(1-m c) s_{m}^{n}+m c . v_{m}{ }^{n+1}
$$

$\mathrm{MC}=$ momentum factor $(\mathrm{o}<\mathrm{mc}<1)$

The boundaries are:

$$
\begin{aligned}
V_{\text {min }} & =X_{\text {min }} \\
V_{\text {max }} & =X_{\text {max }}
\end{aligned}
$$

\section{SSPSO Algorithm}

1. Splitting the search space and divide the particles in each sliced search space.

2. Initialize the total population.

3. For trials $=1: 30$

For iterations $=1:$ maximum iterations

For $\mathrm{K}=1$ : number of particles

For $\mathrm{L}=1:$ number of dimensions 
4. Evaluate the objective function \& fitness of each particle

5. Update the position according to formula (27).

6. Bound the position of particle within the boundaries of the slice.

7. Update the velocity using the formula (25).

8. Bound the velocity of particles within each slice using equations (28) and (29).

9. Find $\mathrm{S}^{*}$-best and fitness of the particle.

10. Check for initialization of each slice is completed, if No go to steps 2-11 otherwise, end.

11. Find g-best fitness, if yes go to 14 , otherwise go to step 4.

12. End the algorithm.

The selected mean of $S^{*} 1, S^{*} 2, S^{*} 3, S^{*} 4$ represents the $\mathrm{S}^{*}$-best value of each slice and the selected mean ' $\mathrm{S}$ ') represents the global best value of each slice. The fitness value of SSPSO for each slice is much better than SPSO.

\section{Simulation Results and Discussions}

The proposed SPSO \& SSPSO algorithms for solving optimal power flows is tested on the IEEE-30 bus test system. Simple-Voltage Stability Index and Fast-Relative Electrical Distance are calculated on the IEEE- 5 bus. The power flow control ability of the power system can be improved with the addition ofSSC device. The improvement in $\mathrm{H}$-index is also observed by adding series compensator in OPF problem with SPSO algorithm. SSC with SPSO is done on the IEEE-30 bus test system.

The Tables 1, 2 gives the parameter settings for SPSO and SSPSO algorithms. Table 3 represents the $\mathrm{H}$-index values at each load bus. The columns correspond to the generator buses numbers and rows correspond to the load bus numbers (Table 4 ).

The load at bus- 3 is $45 \mathrm{MW}$. To meet this load G1 contributes $74.43 \%$ of $45 \mathrm{MW}$ (i.e $45^{*} 0.7443=33.4935 \mathrm{MW}$ ) and G2 contributes $24.49 \%$ of $45 \mathrm{MW}$ (i. e $45^{*} 0.2490$ $=11.205)$. The load at bus -4 is $40 \mathrm{MW}$, for this load G1 gives $79.52 \%$ of $40 \mathrm{MW}$ and G2 gives $19.78 \%$ of $40 \mathrm{MW}$. Similarly the load bus at 5 is $60 \mathrm{MW}$, to meet this load G1 contributes $93.15 \%$ of $60 \mathrm{MW}$ and G2 contributes $6.29 \%$ of 60 MW. (G1-generator 1, G2-generator 2).
Table 1. Optimal parameters for SPSO

\begin{tabular}{|l|c|}
\hline Parameter & SPSO \\
\hline Population size & 20 \\
\hline Number of iteration & 200 \\
\hline Cognitive constant, $\mathrm{c1}$ & 2 \\
\hline Social constant, $\mathrm{c} 2$ & 2 \\
\hline Inertia weight, $\mathrm{w}$ & $0.3-0.9$ \\
\hline
\end{tabular}

Table 2. Optimal parameters for SSPSO

\begin{tabular}{|l|c|}
\hline Parameter & SSPSO \\
\hline Population size & 40 \\
\hline Number of iteration & 150 \\
\hline Cognitive constant, c1 & 2 \\
\hline Social constant, c2 & 2 \\
\hline Inertia weight, $w$ & $0.3-0.9$ \\
\hline momentum factor $(\mathrm{mc})$ & 0.3 \\
\hline
\end{tabular}

Table 3. Simple-Voltage Stability Index for the IEEE-5 bus system

\begin{tabular}{|c|c|}
\hline Load Bus & H-index \\
\hline 3 & 0.0637 \\
\hline 4 & 0.0671 \\
\hline 5 & 0.0772 \\
\hline
\end{tabular}

Table 4. F-RED values for the IEEE - 5 bus system

\begin{tabular}{|c|c|c|c|}
\hline Load Bus & Load (MW) & G1 (\%) & G2 (\%) \\
\hline L3 & 45 & 0.7443 & 0.2490 \\
\hline L4 & 40 & 0.7952 & 0.1978 \\
\hline L5 & 60 & 0.9315 & 0.0629 \\
\hline
\end{tabular}

Cost Characteristics of the IEEE-30 Bus by SPSO and SSPSO

Figure 4 shows the cost characteristics of the IEEE-30 bus by taking number of iterations on $\mathrm{X}$-axis and total generation cost on Y-axis for the first graph. Second graph is the fitness of the cost function which is obtained by taking inverse of the generation cost.

Figure $5 \mathrm{~s}$ the cost characteristics of IEEE-30 bus by using SSPSO algorithm. The generation cost for above system is $800.30(\$ / h r)$ and the loss is 0.0895 p.u. 


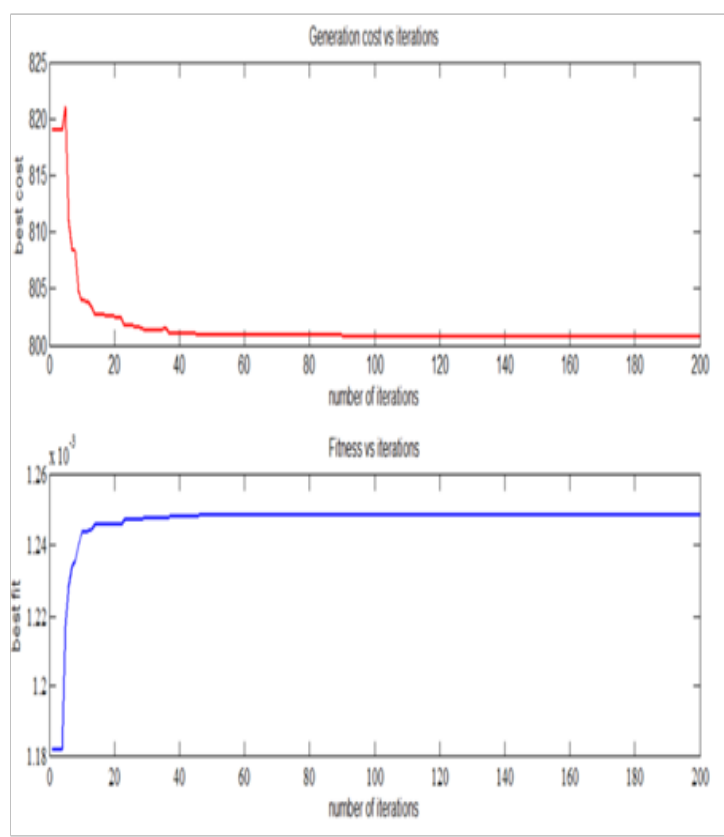

Figure 4. Cost characteristics of the IEEE-30 using SPSO algorithm.
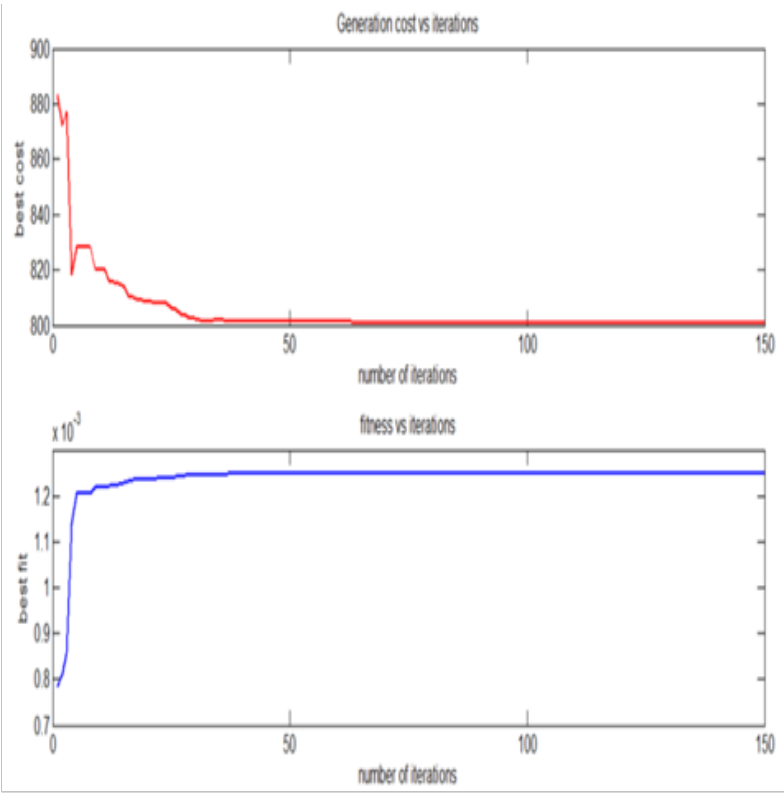

Figure 5. Cost characteristics of the IEEE-30 using SSPSO algorithm.

\section{Comparison between SPSO and SSPSO}

Table 5 shows that the objective functions total generation cost and active power losses are compared by using SPSO and SSPSO. The total generation cost and active power loss are minimized by using SSPSO compared to SPSO (Figure 6).
Table 5. Comparison of SPSO and SSPSO for the IEEE-30 bus system

\begin{tabular}{|l|c|c|}
\hline Objective Function & SPSO algorithm & $\begin{array}{c}\text { SSPSO } \\
\text { algorithm }\end{array}$ \\
\hline Total cost $(\$ / \mathrm{hr})$ & 800.77 & 800.30 \\
\hline Active power loss (p.u) & 0.0972 & 0.0895 \\
\hline
\end{tabular}

The characteristics shows that combined characteristics of SSPSO and SPSO optimization methods. It is clear that SSPSO gives better results compared to SPSO method.

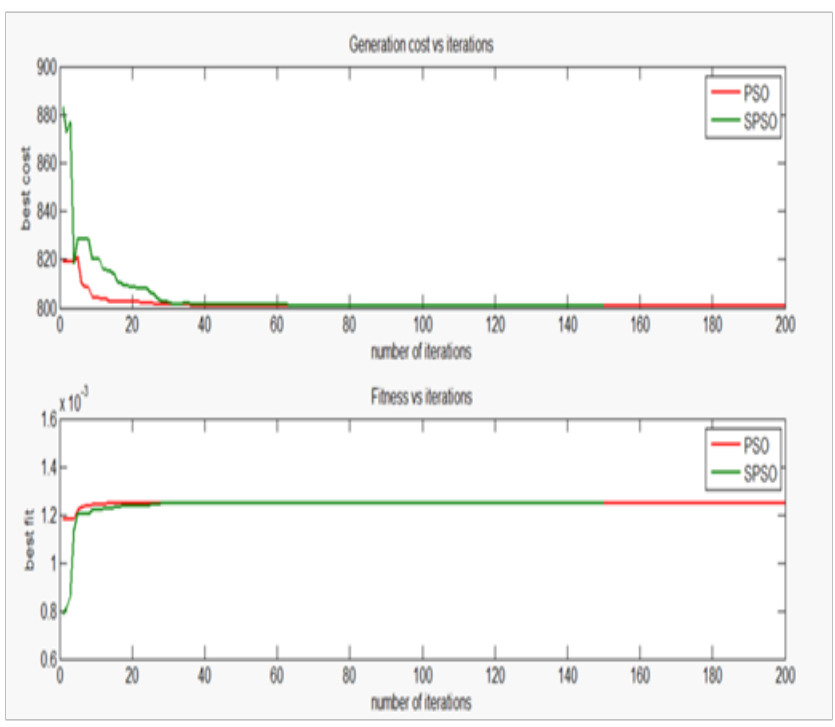

Figure 6. Comparison of Cost characteristics for the IEEE-30 bus system using SSPSO and SPSO.

\section{Results for SPSO with SSC for the IEEE-30 Bwus}

Table 6 describes the voltage stability indices of the IEEE30 bus with the addition of SSC between the buses 9-30. The $\mathrm{H}$-index values are improved with the addition of SSC compared to without SSC (Figure 7).

The Figure 7 shows that the generation cost is the total generation cost plus SSC device cost and second graph shows that inverse of cost function i.e. fitness of the cost function.

Table 6. H-index for the IEEE-30 bus system using SPSO along with SSC

\begin{tabular}{|c|c|c|c|}
\hline Load bus & H-index & Load bus & H-index \\
\hline 7 & 0.0229 & 19 & 0.0987 \\
\hline 8 & 0.0150 & 20 & 0.0946 \\
\hline 9 & 0.0413 & 21 & 0.0871 \\
\hline
\end{tabular}




\begin{tabular}{|c|c|c|c|}
\hline Load bus & H-index & Load bus & H-index \\
\hline 10 & 0.0748 & 22 & 0.0867 \\
\hline 11 & 0.176 & 23 & 0.1012 \\
\hline 12 & 0.0502 & 24 & 0.0987 \\
\hline 13 & 0.0156 & 25 & 0.0955 \\
\hline 14 & 0.0717 & 26 & 0.1096 \\
\hline 15 & 0.0823 & 27 & 0.0868 \\
\hline 16 & 0.0667 & 28 & 0.0208 \\
\hline 17 & 0.0775 & 29 & 0.1160 \\
\hline 18 & 0.947 & 30 & 0.1357 \\
\hline
\end{tabular}

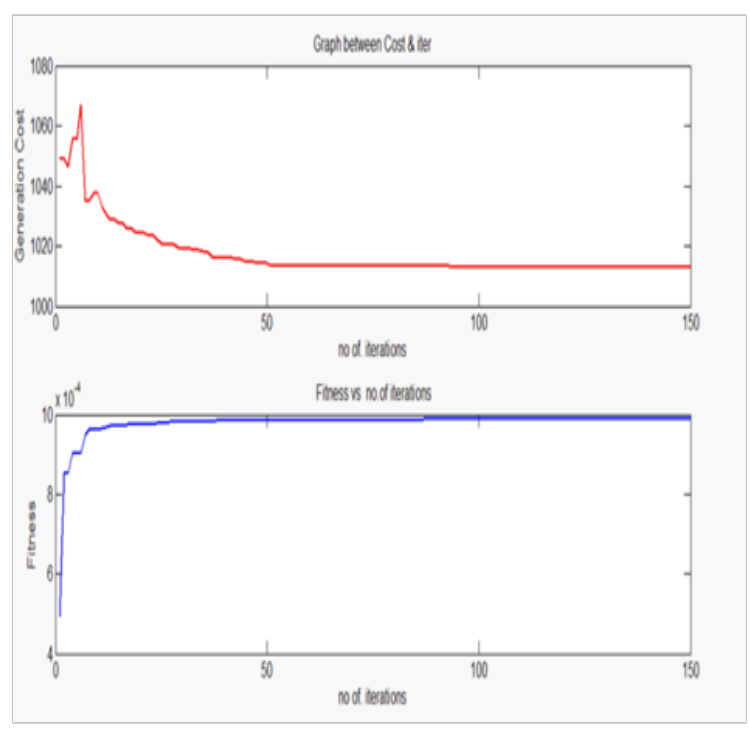

Figure 7. Cost characteristics for the IEEE-30 bus system Using SPSO with SSC.

Table 7 shows the results that are obtained by using SPSO algorithm along with SSC for the IEEE-30 by considering the objective functions as total generation cost and active power loss. The results are obtained by taking all the exact-equality and inequality constraints. Here generation cost represents total generation cost along with the SSC device cost. The active power loss is reduced with the addition of SSC compared to without SSC. H-indexof the system is also improved with the inclusion of SSC.

Table 7. SPSO with SSC results for the IEEE-30 bus system

\begin{tabular}{|l|c|}
\hline Objective function & SPSO with SSC \\
\hline Total generation cost (\$/hr) & 1012.8 \\
\hline Active power loss (p.u) & 0.1138 \\
\hline H- index & 0.1588 \\
\hline
\end{tabular}

\section{Conclusion}

In this study, we implemented SPSO and SSPSO optimization techniques for the solution of power flows under steady conditions and also improvement in the power system was observed with the addition of SSC device on the IEEE-30 bus.H-indexand F-RED are also calculated on the IEEE- 5 bus test systems. The SPSO and SSPSO algorithms give reliable and accurate optimal power flow solutions.

\section{References}

1. Venikov VA, Stroev VA, Idelchick VI, Tarasov VI. Estimation of electric power system steady state stability in load flow calculations. IEEE Transactions on Power Apparatus and Systems. 1975 Jun; 94(3):1034-40. Crossref.

2. Perez-Londono S, Rodriguez LF, Olivar G. A Simplified Voltage Stability Index (SVSI). Electrical Power and Energy Systems. 2014 Dec; 63(2014):806-13.

3. Subramani C, Dash SS, Kumar MJ, Bhaskar MA. Stability index based voltage collapse prediction and contingency analysis. Journal of Electrical Engineering and Technology. 2009; 4(4):438-42. Crossref.

4. Thukaram D, Vyjayanthi C. Relative electrical distance concept for evaluation of network reactive power and loss contributions in a deregulated system. IET Institution of Engineering and Technology Generation, Transmission and Distribution. 2009; 3(11):1000-19. Crossref.

5. Li N, Xu Y, Chen H. FACTS-based power flow control in interconnected power systems. IEEE Transactions on Power Systems. 2000 Feb; 15(1):257-62. Crossref.

6. Hingaroni NG, Gyugyi L. Understanding FACTS: Concepts and technology of flexible AC transmission systems. IEEE Press, New-York; 2000. p. 1-452.

7. Kennedy J, Eberhart M. Particle swarm optimization. Proceedings of IEEE International Conference on Neural Networks; 1995 Dec. Crossref.

8. Abido MA. Optimal power flow using particle swarm optimization. Electrical Power Energy System. 2002; 24(7):563-71. Crossref.

9. Hayeghi H, Shayanfar HA, Shojaei A. An improved PSO based solution for the optimal power flow problems. University of Pitesti - Electronics and Computers Science Scientific Bulletin. 2008; 8(2):99-105.

10. Garg H, Pattnaik SS, Devi S, Bakwad KM, Panigrahiz BK, Das SK. Sliced Particle Swarm Optimization (SPSO): A computationally efficient optimization technique. World Congress on Nature and Biologically Inspired Computing (NABIC); 2009. p. 61-6. Crossref. 